\title{
Liquid Phase Crystallized Silicon - A Holistic Absorber Quality Assessment
}

\author{
Paul Sonntag*1, Matevž Bokalič ${ }^{2}$, Natalie Preissler ${ }^{1 / 3}$, Daniel Amkreutz ${ }^{1}$, Bernd Rech ${ }^{1}$ and Marko Topič² \\ ${ }^{1}$ Institut für Silizium Photovoltaik, Helmholtz Zentrum Berlin für Materialen und Energie GmbH, 12489 Berlin, Germany \\ ${ }^{2}$ Faculty of Electrical Engineering, University of Ljubljana, 1000 Ljubljana, Slovenia
}

${ }^{3} \mathrm{PV}$ comB, Helmholtz Zentrum Berlin für Materialen und Energie GmbH, 12489 Berlin, Germany

Keywords: Liquid Phase Crystallization, silicon solar cells, thin-film, diffusion length, surface recombination, LBIC

*Corresponding author: paul.sonntag@,helmholtz-berlin.de

\begin{abstract}
Liquid Phase Crystallized -Silicon (LPC-Si), the basis for multi-crystalline thin-film solar cells with absorber thicknesses around $15 \mu \mathrm{m}$, is subject to rapid quality improvements. Recently, power conversion efficiencies surpassed $13 \%$. With open circuit voltages above $650 \mathrm{mV}$ and grain sizes of up to $\mathrm{cm}$ in length, LPC-Si morphology and electronic quality has reached mc-Si wafer quality. In this paper, we report on the current status of absorber attributes in LPC-Si cells. To this end, an absorber doping series and an absorber thickness variation are evaluated. It is observed that for state-of-the-art cells, thicknesses over $15 \mu \mathrm{m}$ are not beneficial due to limited diffusion lengths. Lower absorber doping concentrations tend to yield longer intra-grain diffusion lengths $\left(L_{\text {diff }}\right)$ and better passivated grain boundaries, which may be due to lower impurity precipitation. The longer $L_{\text {diff }}$ leads to higher short circuit current densities which over-compensate a decrease in open circuit voltage and fill factor with regards to efficiency. Both front and rear surfaces are sufficiently passivated and at the current status the bulk lifetime has most potential for improvement.
\end{abstract}

\section{INTRODUCTION}

Many approaches are being pursued to bring down material consumption of Si solar cells using the wafering process by alternative fabrication techniques. These alternatives include classical thin-film technologies like amorphous (a-Si) or microcrystalline ( $\mu$-c-Si) Si [1-3], top-down approaches like epitaxial lift-off techniques [4,5] or the liquid phase crystallization (LPC) technology [6]. All of them offer the potential to save material cost while having the advantage to rely on a vast existing knowledge on (crystalline) $\mathrm{Si}$ photovoltaics.

LPC of Si absorbers is a zone-melting procedure using a lineshaped energy source to convert an initially amorphous/nanocrystalline precursor to multi-crystalline material directly on a supporting glass with multi-functional thin $(200 \mathrm{~nm})$ intermediate layers [6]. In contrast to a-Si/ $\mu \mathrm{c}-\mathrm{Si}$ absorbers [7] LPC-Si absorbers can be up to $40 \mu \mathrm{m}$ thick and show grain sizes comparable to multi-crystalline $(\mathrm{mc})-\mathrm{Si}$ wafers $[8,9]$. The technology represents thus a hybrid between wafers and classical bottom-up thin-film technologies. Contrary to that, the elaborate top-down epitaxial lift-off technique uses a high quality mono-crystalline and reusable parent wafer from which absorbers are lifted off and bonded to a substrate [4,5]. Efficiencies of up to $20.6 \%$ were presented on $35 \mu \mathrm{m} \mathrm{Si} \mathrm{[10].}$ With the a-Si/ $\mu \mathrm{c}-\mathrm{Si}$ tandem technology, recently, a $14 \%$ cell was achieved [3]. Present efficiencies on LPC-Si are 13.2\% on below $15 \mu \mathrm{m}$ thick absorbers using an interdigitated back contact (IBC) system and up to $15.9 \%$ on full-emitter test structures [11]. LPC-Si has shown a steep learning curve and progress in recent years, amongst other, thanks to a continuous improvement of the quality of the interface between $\mathrm{Si}$ and the supporting glass by an intermediate layer (IL) stack $[12,13]$ and hydrogen plasma passivation techniques [14]. To push performance even further, a thorough material assessment is necessary to find possible levers for improvement. However, many standard wafer characterization methods do not work due to poor signal/noise ratio (quasi steady state photo conduction, QSSPC) or challenging to evaluate because of substrate effects (photoluminescence, PL). Although it was shown that QSSPC can be measured with under certain circumstances with an altered setup[15], symmetrical lifetime samples can inherently not be processed, because an identical surface treatment as for the buried and inaccessible LPC$\mathrm{Si}$ /supporting glass side cannot be repeated on the other side. Hence, in this publication, we obtain material parameters from illuminated $J-V$-curves of finished devices and compare them to simulations. The studied devices are bifacial so comparing the two illumination configurations (i) through the glass (superstrate) and (ii) from Si side (substrate) conclusions about the glass/LPC-Si interface quality can be drawn. Furthermore, the change in absorber quality of LPC-Si absorbers with varying phosphorous doping concentration is partly quantified using light beam induced current measurements and $J-V$ measurements.

\section{EXPERIMENTAL}

LPC-Si absorbers were fabricated according to the steps in [11]. For the doping series, $15 \mu \mathrm{m}$ and for the thickness variation 14 and $33 \mu \mathrm{m}$ of nominally intrinsic $\mathrm{Si}$ were 
deposited on $1.1 \mathrm{~mm}$ Corning Eagle XG glass using high rate electron beam evaporation. Prior to Si deposition a multifunctional intermediate layer (IL) comprised of $\mathrm{SiO}_{\mathrm{x}} / \mathrm{SiN}_{\mathrm{x}} / \mathrm{SiO}_{\mathrm{x}}$ was deposited as described elsewhere $[12,13,16]$. Amongst others, this IL is crucial for final device performance, since it is the only option to control the passivation and stop impurity diffusion at the glass/LPC-Si interface because later this interface is buried and inaccessible. Subsequent to absorber precursor deposition, a so-called doping layer comprised of a-Si:H(n) was deposited by PECVD to introduce phosphorous (P) dopants. Different final doping concentrations were achieved by varying the phosphine gas flow during deposition. The actual liquid phase crystallization procedure was performed using a line-shaped laser at a wavelength of $808 \mathrm{~nm}$ in vacuum [12]. The absorber is molten entirely, allowing the dopants in the doping layer to disperse homogeneously throughout the absorber. Final doping densities between $2.0 \cdot 10^{16}$ and $7.0 \cdot 10^{17} / \mathrm{cm}^{3}$ were reached this way. The doping densities were determined by sheet resistance measurements according to

$$
N_{D}=\left(e \bar{R}_{\text {sheet }} \mu_{n} d_{0}\right)^{-1},
$$

$\bar{R}_{\text {sheet }}$ being the averaged sheet resistance of measurements on the entire substrate and the elementary charge $e$, initial absorber thickness $d_{0}$ and majority carrier (electron) mobility $\mu_{n}$.

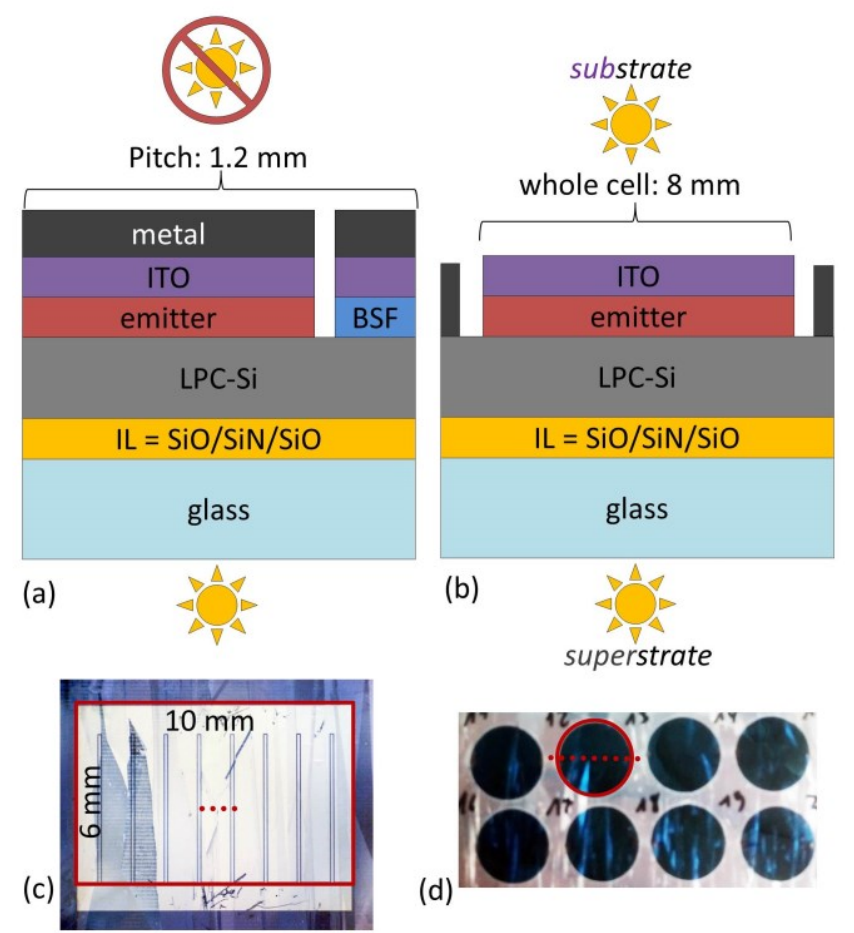

Figure 1: (a) Schematic cross section of one pitch length of an IBC-SHJ cell on LPC-Si on glass. (b) Schematic cross section of a whole quasi cell test structure. (c) Photograph of top-view of an IBC-SHJ (back side). The cell area is indicated by the red full line. The location of the cross section in (a) is indicated by the red dotted line. (d) Top-view of quasi cell test structures in substrate configuration. Red circle indicates cell area, red dotted line the place of the cross section in (b).

The latter was assumed to be $80 \%$ of the mobilities of the Masetti model [17] for mono-crystalline Si to account for the poly-crystalline structure. This is in accordance with earlier findings $[8,9,14]$. An error for the resulting $N_{D}$ was calculated from the standard deviation of all $R_{\text {sheet }}$ measurements. To augment absorber quality a hydrogen plasma treatment $[12,14]$ was performed prior to contact system processing. Finally, interdigitated back contact (IBC) cells using silicon heterojunctions (SHJ) were fabricated on the absorbers for the doping series (Fig. $1(\mathrm{a}, \mathrm{c})$ ). Bifacial circular cell test structures (quasi cells, Fig. 1(b,d)) having the lowest doping concentration of the doping series were processed on the absorbers for the thickness variation. Both will be introduced in detail in the following.

\section{A. Interdigitated Back Contact Cells}

A cross section of an IBC-SHJ unit cell (pitch) is shown schematically in Fig. 1(a) and a photograph of the back (metal) side of a whole cell is depicted in Fig. 1(c). The red dotted line indicates the length of the cross section in (a). The red full rectangle represents the area of the cell (emitter busbar is also counted as cell area). The whole fabrication process in elaborate detail can be found in [11]. In total 44 cells were processed on 4 substrates of doping densities of $2.0 \cdot 10^{16}$, $1.4 \cdot 10^{17}, 1.7 \cdot 10^{17}$ and $7.0 \cdot 10^{17} / \mathrm{cm}^{3}$. The contact system was co-processed for all cells in the same run and all cells received identical wet chemistry treatment.

\section{B. Quasi Cell Test Structures}

A schematic cross section of a quasi cell test structure and a top-view photograph can be seen in Fig. 1(b) and (d), respectively. The sun-pictographs indicate that operation in substrate (front-emitter) and superstrate (rear-emitter) configuration is possible. The cell diameter is $8 \mathrm{~mm}$ corresponding to an emitter area of $\sim 0.5 \mathrm{~cm}^{2}$. The absorber contact is not passivated and lies outside the defined cell area (cp. red circle in (d)). On one hand the cell has an inherently low fill factor $(F F)$ due to a high base series resistance. On the other hand, it is comparably simple to fabricate, fully bifacial and useful for screening short circuit current density $\left(J_{S C}\right)$ and open circuit voltage $\left(V_{O C}\right)$. The surfaces of the $N_{D}=2 \cdot 10^{16} / \mathrm{cm}^{3}$ absorbers are first cleaned and an a-Si:H(i/p) emitter is formed using low-temperature PECVD. Subsequently, $80 \mathrm{~nm}$ ITO is RF-sputtered from a ceramic target. The cell area is defined by an adhesive masking tape with circular shape of $4 \mathrm{~mm}$ radius made of Kapton ${ }^{\circledR}$ polyimide film with silicone adhesive by Max Steier GmbH \& Co. KG. Subsequent to mask-taping the ITO surface, the samples were etched for $10 \mathrm{~s}$ in $20 \% \mathrm{HCl}$ to 
remove the ITO surrounding the Kapton ${ }^{\circledR}$ dots. In the second etch step of 15-20 s, in a mixture of nitric, phosphorous, and hydrofluoric acid, possible remains of the ITO are removed and the a-Si:H(i/p) emitter is dissolved. After the HF-dip, the samples were thermally coated with $30 \mathrm{~nm}$ Ti and $1000 \mathrm{~nm}$ Al. The Kapton ${ }^{\circledR}$ dots are temperature resistant up to $270^{\circ} \mathrm{C}$ and not affected during all the acidic treatments. Finally, the dots were removed exposing the finished quasi cell test structures depicted in Figure 1(d). Since all the etching solutions used creep also (on a $\mu \mathrm{m}$ scale) underneath the Kapton ${ }^{\circledR}$ dots an isolation gap is inherently formed during the process between the ITO contact which serves as p-type contact, and the Ti/Al absorber (n-type) contact.

\section{Methods}

Illuminated $J-V$ curves were recorded with a dual source solar simulator by Wacom having AAA characteristics [18].

LBIC measurements were conducted using a self-built setup at the University of Ljubljana with a laser of $638 \mathrm{~nm}$ wavelength, a spot size below $10 \mu \mathrm{m}$ and an $x, y, z$ stage with minimum step width of $1 \mu \mathrm{m}$ [19].

Simulations were performed using ASPIN3, a numerical 2D solar cell simulation software, developed at the University of Ljubljana [20].

\section{RESULTS}

\section{A. Doping Series}

The main parameters obtained from the recorded illuminated $J$ - $V$-curves of all the 44 fabricated cells are shown in Figure 2 except for 3 outliers. The fluctuation in performance leading to the standard deviation of measurements shown as error bars can be attributed to the inhomogeneous mc-Si-like morphology of LPC-Si. We observe a strong decrease of $J_{S C}$ with higher doping (red) and a slight increase of $V_{O C}$ (blue) that seems to decrease again for $N_{D}=7.0 \cdot 10^{17} / \mathrm{cm}^{3}$. The $F F$ tends to increase with doping (orange), while overall conversion efficiency drops steadily (green), following the trend of $J_{S C}$. Please note that to reach current densities above $30 \mathrm{~mA} / \mathrm{cm}^{2}$ as in $[21,22]$ a light trapping foil is usually additionally placed on the front glass which has been omitted here. Black circles and triangles are calculated $V_{O C}$ values that will be explained in the discussion section.

Light beam induced current (LBIC) measurements of the best cells with $N_{D}=2.0 \cdot 10^{16}, 1.7 \cdot 10^{17}$ and $7.0 \cdot 10^{17} / \mathrm{cm}^{3}$ were recorded to investigate grain boundary (GB) behavior and evaluate diffusion lengths using a method presented in [23]. The method uses LBIC line-scans to measure the collection signal drop at the absorber contact, where no minority carriers are collected. A measure for this signal drop is the effective diffusion length $L_{\text {diff }}$ (taking bulk and interface effects into account) which is obtained by a double exponential fit of the line-scans [23]. The cells on the substrate containing $N_{D}=$ $1.4 \cdot 10^{17} / \mathrm{cm}^{3}$ were omitted, since it is very close to the one of $1.7 \cdot 10^{17} / \mathrm{cm}^{3}$. The complete LBIC 2D collection maps are shown in Figure $3(\mathrm{a}-\mathrm{c})$. The images were all scaled from 0 to the median of all pixel values. All areas with values above the median appear white. The absorber contacts appear black, since the minority charge carriers generated in this region have to travel laterally to the minority contacts ("electrical shading"). Other areas of reduced collection are due to multicrystalline nature of LPC-Si (GBs and dislocations). It can be seen that the GBs of the cell with $N_{D}=7.0 \cdot 10^{17} / \mathrm{cm}^{3}$ are substantially better visible due to a higher signal drop caused by higher recombination than the other two. Diffusion lengths were determined at various places on the cells inside grains at dislocation free (white in the images) areas using line-scans with $5 \mu \mathrm{m}$ steps (not shown). The fitted diffusion lengths were plotted versus $N_{D}$ in Figure 3(d). With rising doping concentration the measured diffusion length drops as well as its standard deviation (cp. error bars). The behavior of cells with varying absorber doping concentration was only determined experimentally because for a predictive theoretical simulation approach too many parameters are unknown.

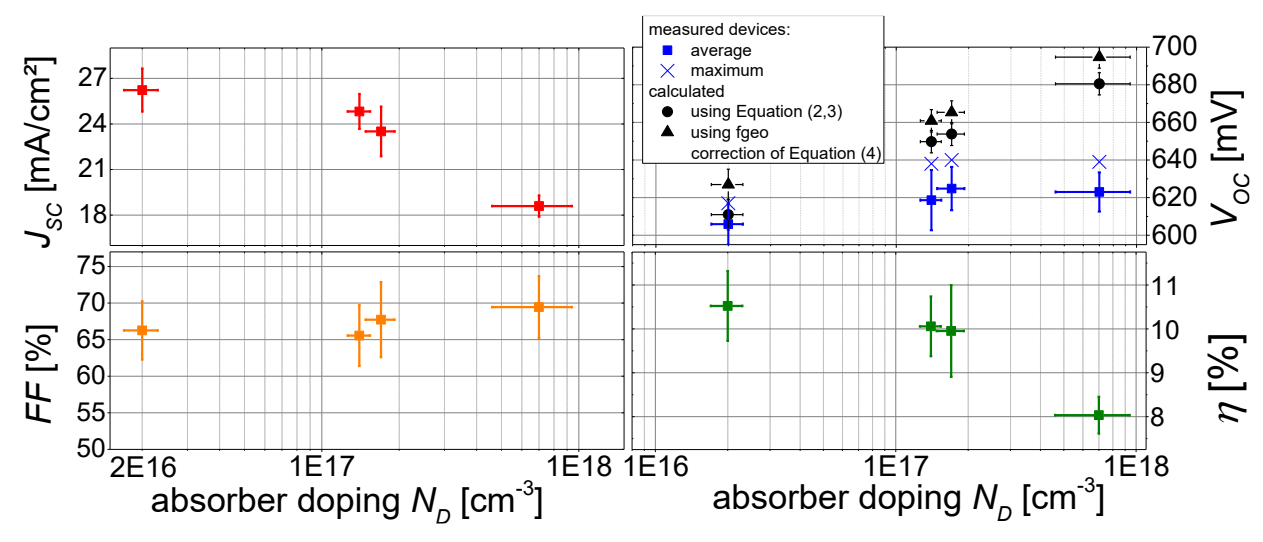


Figure 2: Squares: Main solar cell parameters obtained from measuring illuminated J-V curves of IBC-SHJ cells (in substrate configuration) plotted versus the absorber doping concentration $N_{D}$. Errors in y-direction are standard deviations of mean of the respective parameter, errors in $x$-direction stem from standard deviation of sheet resistance measurements used to determine the absorber doping. Circles: VOC values calculated using Equation (2,3), mobility assumed to be $80 \%$ of mono-crystalline $S i, L_{d i f f}$ values from Table I were used. Triangles: $V_{O C}$ values calculated using the additional correction of Equation (4).

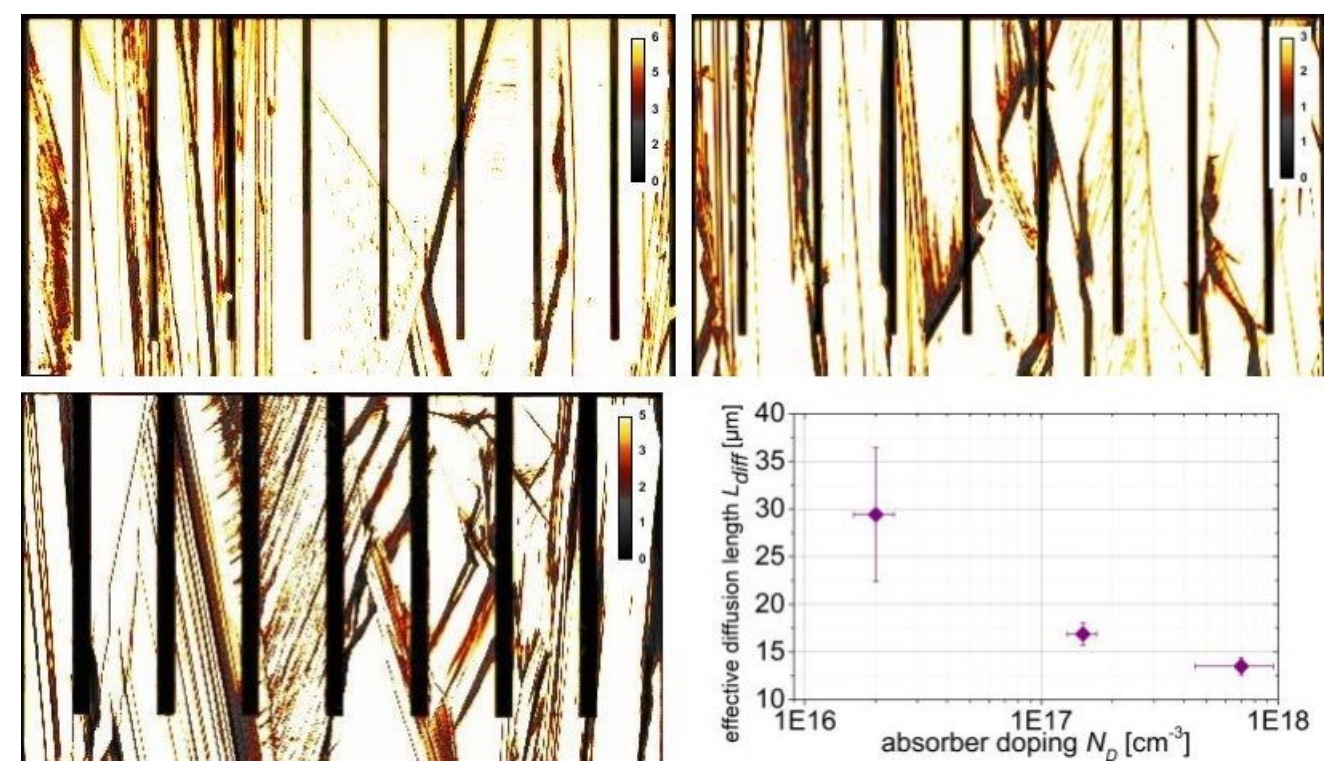

Figure 3: (a)-(c): complete LBIC maps of the best cells of different doping concentrations. (a) $N_{D}=2.0 \cdot 10^{16} / \mathrm{cm}^{3}$ (b) $N_{D}=$ $1.7 \cdot 10^{17} / \mathrm{cm}^{3}$ (c) $N_{D}=7.0 \cdot 10^{17} / \mathrm{cm}^{3}$. Images are scaled from 0 to the median of all pixel values of the respective cell. All areas with values above the median appear white. (d): Diffusion lengths determined at areas inside grains at an absorber contact strip of (a)-(c), values are listed in Table I.

Among these parameters influencing SRH lifetime are impurities $(\mathrm{O}, \mathrm{N}, \mathrm{C}$, metals), their type, the density and structure of defects, the absorber doping itself, the ratio of actively contributing dopants, grain size and GB properties [24].

\section{B. Thickness Variation}

Illuminated $J-V$ curves were measured in sub- and superstrate configuration for both $14 \mu \mathrm{m}$ and $33 \mu \mathrm{m}$ thick bifacial quasi cells with $N_{D}=2 \cdot 10^{16} / \mathrm{cm}^{3}$. The obtained mean values and standard deviations of $J_{S C}$ and $V_{O C}$ of all non-shunted cells were plotted as orange stars in Figure 4; the maximum values are indicated by orange lines. Note that $F F$ and efficiency are not included because quasi cells have an inherently high base resistance so no useful information can be drawn from these values.

The devices' $V_{O C}$ and $J_{S C}$ were also simulated using ASPIN3 to compare them with the measured ones. To this end, a structure representing the geometries of a quasi cell test structure was implemented into ASPIN3. The optics were adapted to real device optics measured via UV/VIS spectroscopy (not shown). The simulation domain was half the cross section in Figure 1. In the simulation, the absorber contact has to be part of the domain, but was made sufficiently small. An isolation gap of $60 \mu \mathrm{m}$ was assumed accounting for the long cumulative ITO etching times in three consecutive acidic solutions. Mobilities of the LPC-Si with an absorber doping of $N_{D}=2 \cdot 10^{16} / \mathrm{cm}^{3}$ were set fixed to $\mu_{n}=859 \mathrm{~cm}^{2} / \mathrm{Vs}$ and $\mu_{p}=244 \mathrm{~cm}^{2} / \mathrm{Vs}$ for electrons and holes, respectively, which corresponds to $80 \%$ of the mobilites obtained from the Masetti model [17]. The LPC-Si(n)/a-Si:H(i/p) interface was assumed as non-limiting and negligible in terms of its surface recombination due to the high quality passivated heterojunctions we used [25]. The parameters varied during the simulations were the effective surface recombination velocity of the LPC-Si/glass interface $S_{\text {eff }}$ (in $\mathrm{cm} / \mathrm{s}$ ) and the minority bulk SRH lifetime $\tau_{p}$ (in $\mu \mathrm{s}$ ). To check the general validity of the approach, measured and simulated quantum efficiencies were compared (not shown). They resulted in having the same features like humps, bumps and general slope. Subsequently, $J-V$ curves were simulated with a glass/LPC-Si interface recombination velocity of $200 \mathrm{~cm} / \mathrm{s}$ and $800 \mathrm{~cm} / \mathrm{s}$. They are plotted in the first and second columns of each separate section in Figure 4, respectively. The lifetime $\tau_{p}$ was varied from $0.5 \mu \mathrm{s}$ to $20 \mu \mathrm{s}$ in various steps. For the $800 \mathrm{~cm} / \mathrm{s}$ version, only the $20 \mu \mathrm{s}$ result is plotted. In Figure 4(a) we can see that while the current for the thin cells is equal for both illumination types, the current for the thick cells is reduced by $7 \mathrm{~mA} / \mathrm{cm}^{2}$. Yet, in substrate configuration, i.e. front emitter operation, the current is higher than for the thin cells. For a simulated $S_{\text {eff }}$ of $200 \mathrm{~cm} / \mathrm{s}$ for the thin $(14 \mu \mathrm{m})$ cells the 
behavior of a constant current independent of illumination type, can only be adequately imitated by lifetimes above $2 \mu \mathrm{s}$. For $800 \mathrm{~cm} / \mathrm{s}$ this is not even possible with a lifetime of $20 \mu \mathrm{s}$. For the thick cells the simulated current shows almost no breakdown at all for $20 \mu \mathrm{s}$ and $200 \mathrm{~cm} / \mathrm{s}$. In Figure 4(b) we can see that the overall measured $V_{O C}$ trend can be mimicked quite well by lifetimes of $0.5 \mu \mathrm{s}-2 \mu \mathrm{s}$ and $200 \mathrm{~cm} / \mathrm{s}$, but the simulation fails to represent the measurement for higher lifetimes and also for a lifetime of $20 \mu \mathrm{s}$ and $800 \mathrm{~cm} / \mathrm{s}$ (partly reversed trend).
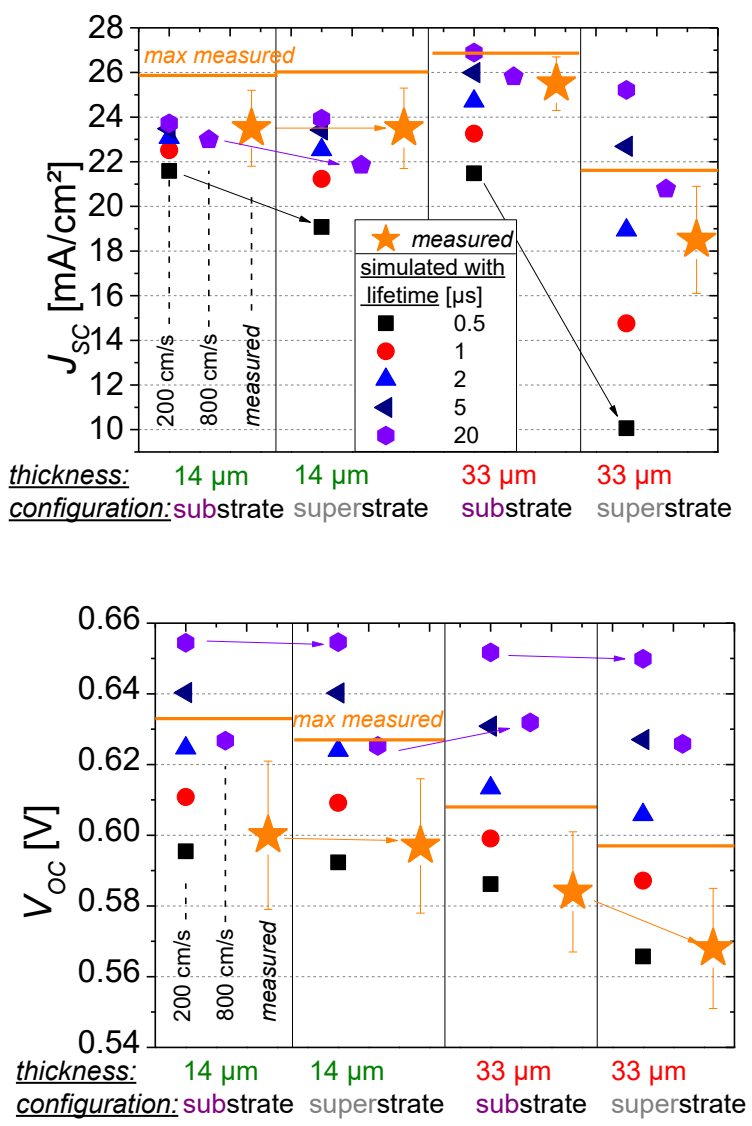

Figure 4: Mean values and standard deviations of measured $J_{S C}(a)$ and $V_{O C}(b)$ for thin and thick cells in superstrate and substrate configurations are indicated as orange stars. The maximum values are represented by orange lines. The devices were simulated with lifetimes between 0.5 and $20 \mu$ s and glass $/ L P C-S i$ interface recombination velocities of $200 \mathrm{~cm} / \mathrm{s}$ (first values in each section) and having a lifetime of $20 \mu \mathrm{s}$ and $800 \mathrm{~cm} / \mathrm{s}$ (second value in each section).

\section{DISCUSSION}

The general trends observed for the doping concentration series are as expected from general theory. A higher $N_{D}$ results in lower hole mobilities $\mu_{p}$ [17] and Auger and SRH recombination begin to rise which all negatively affect the minority diffusion length $L_{\text {diff }}$. This has a primary impact on $J_{S C}$, which can be observed clearly in Figure 2. A direct proof of the decreasing diffusion lengths are also the measured values depicted in Figure 3(d) and again listed in Table I. Despite this increase in absorber recombination, the $V_{O C}$ rises with increasing $N_{D}$, solely due to the higher absorber doping itself. This can be understood by looking at how the $V_{O C}$ depends on $J_{S C}, L_{d i f f}, \mu_{p}$ and $N_{D}$. From literature it is known that the open-circuit voltage follows

$$
V_{O C} \propto V_{t h} \ln \left(\frac{J_{S C}}{J_{0}}\right),
$$

with the thermal voltage $V_{t h}=k T / e$ (elementary charge $e$, Boltzman constant $k$ and absolute temperature $T$ ), the short circuit current density $J_{S C}$, and the saturation current density $J_{0}$. The latter is a function of all other sought parameters and can be expressed as

$$
J_{0}=V_{t h} \cdot e \cdot n_{i}^{2} \cdot\left(\frac{\mu_{p}}{L_{\text {diff }} \cdot N_{D}}\right),
$$

with the intrinsic carrier concentration $n_{i}{ }^{2}$. Until a doping concentration of $1.7 \cdot 10^{17} / \mathrm{cm}^{3} V_{O C}$ rises (see Figure 2). For higher $N_{D}$, the losses in $L_{d i f f}$ (Equation 3) and $J_{S C}$ (Equation 2) seem to start over-compensating the impact of the doping itself and $V_{O C}$ sinks again slightly. However, this trend cannot be reproduced if we assume the diffusion lengths found by LBIC (Figure 3, Table I) and insert the values together with literature data for $\mathrm{n}_{\mathrm{i}}^{2}, V_{t h}$ and $e$ in Eqs. $(2,3)$ and plot them together with the measured $V_{O C}$ in Figure 2 (see black circles). Neither does the trend change if we take a correction factor for $J_{0}$ in Equation 2 into account; the so-called geometry factor $\left(f_{\text {geo }}\right)$ [24]. It is a correction factor for the case of a finite absorber thickness and if the emitter-sided interface is not recombination active. Hence, it can be applied for the investigated IBC-SHJ cells, since the emitter-side is a-Si:H passivated [25]. The equation for $J_{0}$ under these assumptions reads

$J_{0}=\frac{e n_{i}^{2} D}{N_{D} L_{b u l k}} \underbrace{\left(\frac{\cosh \left(W_{\text {abs }} / L_{\text {bulk }}\right)+D /\left(L_{\text {bulk }} S_{\text {eff }}\right) \sinh \left(W_{\text {abs }} / L_{\text {bulk }}\right)}{D /\left(L_{\text {bulk }} S_{\text {eff }}\right) \cosh \left(W_{\text {abs }} / L_{\text {bulk }}\right)+\sinh \left(W_{a b s} / L_{\text {bulk }}\right)}\right)}_{f_{\text {go }}}$

with the surface recombination velocity $S_{\text {eff }}$ (glass/LPC-Si), the diffusion coefficient $D$ of the minority carriers in the absorber and its thickness $W_{a b s}$. An $S_{\text {eff }}$ of $200 \mathrm{~cm} / \mathrm{s}$ was chosen for estimating $f_{\text {geo }}$ which is in accordance with results from the thickness variation on the quasi cell test structures that will be discussed later on and the using the diffusion coefficient

$$
D=V_{t h} \mu_{p}
$$


The bulk diffusion length $L_{b u l k}$ was calculated from the effective diffusion lengths $L_{\text {diff }}$ in Table I and the assumed $S_{\text {eff }}$. We can see that the trend for the calculated $V_{O C}$ in Figure 2 including $f_{\text {geo }}$ (black triangles) does still not match the measurements. Either the assumed $80 \%$ of the monocrystalline Si mobilities are not correct for higher $N_{D}$ or the effective diffusion length. Since the latter was only measured inside grains at places of high current collection (cp. white areas in Figure $3(\mathrm{a}-\mathrm{c})$ ) and not at dislocations and grain boundaries and thus does not represent an average or global diffusion length the behavior can be explained. To make the trend match the measurement, a global diffusion length of 3$4 \mu \mathrm{m}$ instead of the locally determined $13 \pm 1 \mu \mathrm{m}$ would be necessary for the highest $N_{D}$ of $7.0 \cdot 10^{17} / \mathrm{cm}^{3}$. The difference between measurement and calculations (Figure 2, blue and black) is much less for the lowest doping concentration,

\section{TABLE I}

\section{LBIC EVALUATION CHART FROM IMAGES DISPLAYED IN} FIGURE 3

\begin{tabular}{|c|c|c|c|}
\hline $\begin{array}{c}\text { Sample dopant } \\
\text { concentration }\left[\mathrm{cm}^{-3}\right]\end{array}$ & $2.0 \cdot 10^{16}$ & $1.7 \cdot 10^{17}$ & $7.0 \cdot 10^{17}$ \\
\hline Diffusion length $[\mu \mathrm{m}]$ & $29 \pm 7$ & $17 \pm 1$ & $13 \pm 1$ \\
\hline Effective lifetime $[\mu \mathrm{s}]$ & $1.3 \pm 0.6$ & $0.5 \pm 0.1$ & $0.3 \pm 0.1$ \\
\hline $\mathrm{GB} /$ dislocation losses $[\%]$ & 7 & 11 & 15 \\
\hline average $J_{S C}\left[\mathrm{~mA} / \mathrm{cm}^{2}\right]$ & $26 \pm 1$ & $24 \pm 2$ & $19 \pm 1$ \\
\hline
\end{tabular}

indicating that here global and locally measured diffusion length lie closer together. This can actually be explained by the 2D LBIC scans in Figure 3 (a-c) because the images at different doping concentrations show a different impact of GB/dislocations that influence the global $L_{\text {diff. Note that }}$. this analytical analysis is rather crude, since we omitted the influence that a different diode ideality factor could have on the trends so the results should be regarded with care. Determining also statistics on the ideality of all the measured cells would exceed the scope of this work.

Next, it was tried to quantify the losses at GB/dislocations as already described in [11] by deriving a current collection loss percentage due to absorber inhomogeneities. Losses of 7, 11 and $15 \%$ were measured for $2 \cdot 10^{16}, 1.7 \cdot 10^{17}$ and $7 \cdot 10^{17} / \mathrm{cm}^{3}$ absorbers, respectively, and are listed in Table I. Although this method may not be very accurate, it shows the trends quite well and furthermore the loss percentage of $11 \%$ for $N_{D}=$ $1.7 \cdot 10^{17} / \mathrm{cm}^{3}$ corresponds exactly to the one obtained for a cell with a similar absorber doping investigated in [11]. We can see in the table that not only does $L_{\text {diff }}$ inside grains decrease but also the amount of losses due to $\mathrm{GB} /$ dislocations increases with higher doping concentration. This leads to the conclusion that a higher doping concentration can be responsible for a higher $\mathrm{GB} /$ dislocation density and/or greater impact of said defects in LPC-Si. The higher defect density affects $V_{O C}$, since dislocations are more difficult to passivate (both inside the absorber bulk, and in terms of the interface towards the a$\mathrm{Si}: \mathrm{H}$ ), as well as $J_{S C}$ (reduced LBIC collection signal). We can see the effect on $V_{O C}$ directly when we look at the trends for measured and calculated $V_{O C}$ in Figure 2 (blue and black, respectively). If we assume the locally measured $L_{d i f f}$ as the overall (global) one, we get the wrong trend. The local $L_{\text {diff }}$ over-estimates the measured $V_{O C}$ more for higher $N_{D}$, where also the impact/losses of $\mathrm{GB} /$ dislocations are higher. Why the impact of defects is increased with $N_{D}$ is subject to speculation but might be related to the greater amount of foreign/impurity atoms (dopant atoms being counted as impurity atoms). It is known that phosphorus (P) segregates at GBs [26,27]. Mandurah et al. [27] stated that the fraction of dopant atoms segregating at GBs is inversely proportional to the size of the grains. For higher doping concentrations in LPC-Si material this would mean that (i) since more $\mathrm{P}$ is present, more $\mathrm{P}$ also segregates at the GBs, and (ii) if the grains themselves are also smaller, an even higher fraction of $\mathrm{P}$ segregates there creating a positive feedback. The $\mathrm{P}$ at the GB influences electrical conduction [27] and all kinds of other impurities precipitate there (which is used in the $\mathrm{P}$ diffusion gettering technique $[28])$.

Since all samples were co-processed and received an identical wet chemical treatment, the change in $F F$ between the samples is attributed to a difference in base doping (cp. Figure 2). This is due to lower sheet resistances leading to a lower series resistance contribution from the bulk. Between the two outer extreme doping densities, an average $F F$ difference of more than $3 \%$ abs was measured. In summary, the gain in $V_{O C}$ and $F F$ towards higher doping concentrations cannot over-compensate the reduction in $J_{S C}$ so that the overall power conversion efficiency shown in Figure 2 is highest for $N_{D}=2 \cdot 10^{16} / \mathrm{cm}^{3}$.

The observed trends from the thickness variation i.e. quasi cell test structures of $14 \mu \mathrm{m}$ (thin) and $33 \mu \mathrm{m}$ (thick) LPC-Si absorbers in Figure 4 allow the following conclusions. Since the measured $J_{S C}$ (orange stars) in sub- and superstrate configuration for the thin absorbers is equally high, (i) the diffusion length is sufficiently long, (ii) both front and back side are sufficiently passivated so that neither of them is limiting. The current for the thick absorbers in substrate configuration is higher, since more photons can be absorbed due to their longer path through the absorber. Their breakdown in $J_{S C}$ in superstrate configuration can be due to (i) insufficient bulk lifetime, (ii) highly recombination active glass/LPC-Si interface. The latter is unlikely to happen since both thin and thick absorber samples received the same intermediate layer (IL) between glass and LPC-Si. The only difference was the energy input of the line-source used for crystallization, which might have altered the interface quality. From a macroscopic 
view on the absorber and from the general level of $V_{O C}$ and $J_{S C}$ (in substrate) we can, however, conclude that differences from the process have a negligible impact on bulk and interface quality.

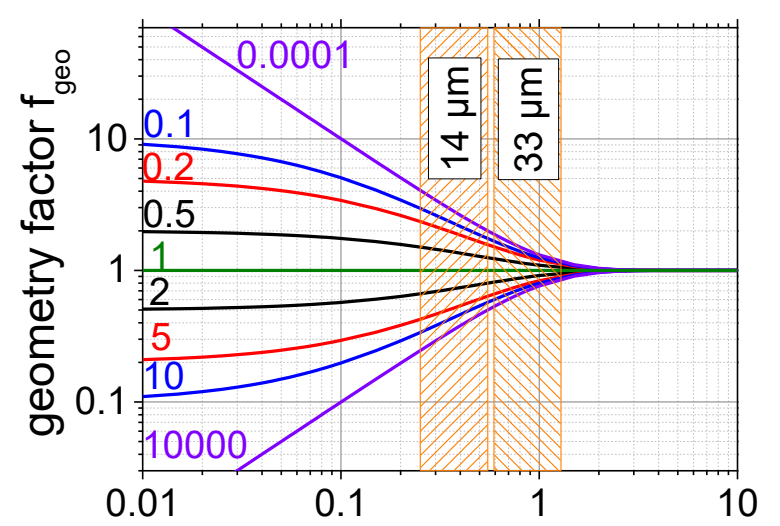

absorber thickness / bulk diffusion length

Figure 5: $f_{\text {geo }}$ as a function of $W_{\text {abs }} / L_{\text {bulk }}$ for representative values of $D /\left(L_{b u l k} S_{\text {eff }}\right)$. The ranges for $W_{\text {abs }} / L_{\text {bulk }}$ ratios for the absorbers of $14 \mu \mathrm{m}$ and $33 \mu \mathrm{m}$ are added as orange hatched areas. Lower $W_{\text {abs }} / L_{\text {bulk-ratio means more influence of the front }}$ interface quality. Higher $f_{\text {geo }}$ indicates bulk limitation, lower indicates interface limitation. Note the double log-axis.

Due to this, all the following conclusions drawn will be under the assumption that the material and interface quality of both thin and thick absorbers is the same and is thus also the same for the IBC-SHJ cells of the absorber doping series with the lowest $N_{D}$, namely $2.0 \cdot 10^{16} / \mathrm{cm}^{3}$. The fact that the $V_{O C}$ for the thick absorbers is slightly smaller is conforming to the fact that the $V_{O C}$ is also a function of absorber thickness. From the general picture of the $J_{S C}$ trend we can conclude that the effective diffusion length for the thin absorbers is long enough for efficient superstrate operation, while for the thick absorbers it is insufficient. This confirms the findings of the doping series (Table I).

To compare the measurements to the simulation, we will focus more on observed trends, than on absolute values due to possible measurement errors. The trends of a breakdown in $J_{S C}$ for the thick absorber in superstrate configuration cannot be imitated correctly by a bulk lifetime of $20 \mu \mathrm{s}$ and $200 \mathrm{~cm} / \mathrm{s}$ and furthermore the simulated $V_{O C}$ are too high (Figure 4). An $S_{\text {eff }}$ of $800 \mathrm{~cm} / \mathrm{s}$ fails to imitate the constant $J_{S C}$ for $14 \mu \mathrm{m}$ in both configurations, even at $20 \mu \mathrm{s}$ where the current saturates. Hence, $S_{\text {eff }}$ has to be smaller than $800 \mathrm{~cm} / \mathrm{s}$, and $\tau_{p}$ smaller than $20 \mu \mathrm{s}$. Furthermore, a lifetime $\tau_{p}=0.5 \mu$ s yields a too strong decrease of $J_{S C}$ from sub- to superstrate so that we can safely assume the lifetime to be greater than $0.5 \mu \mathrm{s}$. The corresponding $L_{\text {diff }}$ regime would be $24-43 \mu \mathrm{m}$ for the thin, and $25-49 \mu \mathrm{m}$ for the thick absorbers. This is in-line with the measurement results for the $2 \cdot 10^{16} / \mathrm{cm}^{3}$ cells from the doping series (cp. Figure 3(d), Table I). One of the objectives of the thickness variation was to find out whether LPC-Si bulk or the buried and inaccessible glass/-LPC-Si interface would be limiting the performance.

To this end, we will, again, make use of $f_{\text {geo }}$ which also serves as a figure of merit to check whether bulk or interface have poorer passivation properties. The geometry factor is only determined by the two ratios (i) $W_{a b s} / L_{b u l k}$ and (ii) $D /\left(L_{b u l k} S_{e f f}\right)$, and the bulk diffusion $L_{b u l k}$ length being

$$
L_{b u l k}=\sqrt{D \tau_{p}}
$$

including $\tau_{p}$. This means $f_{\text {geo }}$ directly depends on both parameters that were varied in the simulations $\left(\tau_{p}\right.$ and $\left.S_{\text {eff }}\right)$. In Figure $5 f_{\text {geo }}$ is plotted as a function of $W_{a b s} / L_{b u l k}$ for several selected $D /\left(L_{\text {bulk }} S_{\text {eff }}\right)$ ratios. A similar representation of this function can be found in [24]. For $L_{b u l k} \geqslant 2 W_{a b s}$, the function collapses and $f_{\text {geo }}$ becomes independent of $S_{\text {eff }}$ at the LPC$\mathrm{Si} /$ glass interface. For lower ratio values, two cases can be identified.

(i) for $D /\left(L_{b u l k} S_{e f f}\right)>1$ the bulk has a higher recombination activity than the surface,

(ii) for $D /\left(L_{b u l k} S_{\text {eff }}\right)<1$ the LPC-Si/glass interface is limiting $J_{0}$ and hence increasing the absorber thickness would be beneficial for performance. For reference the $W_{a b s} / L_{b u l k}$ regime corresponding to a possible parameter space (1-5 $\mu \mathrm{s})$ determined by comparing simulation and measurement in Figure 5 is added in the graph as orange hatched areas. The area for the thick absorber is shifted towards higher $W_{a b s} / L_{b u l k}$, where the influence of $S_{\text {eff }}$ on $f_{\text {geo }}$ and hence also on $J_{0}$ is smaller. If we insert also the derived $S_{\text {eff }}$ of $200 \mathrm{~cm} / \mathrm{s}$ to compute $D /\left(L_{b u l k} S_{\text {eff }}\right)$ values for thin and thick absorbers we get values between 5 and 13, corresponding to the lower red and blue curve in the graph. The orange hatched area between these two curves is our estimate for $f_{\text {geo }}$. It tells us that the bulk material quality is limiting the performance for the investigated quasi cell test structures and thus also for the lowest doping concentration of the IBC-SHJ doping series. Consequently, it would be a futile approach to increase cell efficiency of the advanced IBC-SHJ cells (Figure 1(a)) by increasing the absorber thickness. The result of a "bulk limitation" is not sensitive to changes in both $\tau_{p}\left(\right.$ or $\left.L_{b u l k}\right)$ and $S_{\text {eff. }}$ The ratio $D /\left(L_{b u l k} S_{\text {eff }}\right)$ becomes smaller than 1 (case (ii)) only if $S_{\text {eff }} \geqslant 1200 \mathrm{~cm} / \mathrm{s}$ assuming a bulk lifetime of $5 \mu \mathrm{s}$ or if $S_{\text {eff }} \geqslant 600 \mathrm{~cm} / \mathrm{s}$ for a bulk lifetime of $20 \mu \mathrm{s}$. Both cases have been excluded by the previous investigation of comparing simulation with experiment in Figure 4. In superstrate configuration, $S_{\text {eff }}$ plays a bigger role than in substrate. It may weaken the statement that the bulk limits the performance for superstrate to some extent, but is still valid for the thin $14 \mu \mathrm{m}$, since not only the bulk diffusion length $\left(L_{\text {bulk }}\right)$, but also the effective diffusion length $\left(L_{d i f f}\right)$ are longer than two times the absorber thickness $\left(W_{a b s}\right)$. The fact that according to this result, the current LPC-Si material is bulk limited, does not imply that a change in surface quality will have no influence. It 
indicates that the cell performance is much more sensitive to changes in the bulk lifetime and that increasing the bulk lifetime offers a higher potential to improve cell performance than increasing surface quality.

\section{CONCLUSIONS}

In this paper we have investigated and partly quantified the behavior of n-type liquid phase crystallized $\mathrm{Si}$ solar cell absorbers depending on absorber doping and absorber thickness. To this end, one IBC-SHJ cell doping series spanning almost two magnitudes of absorber doping and quasi cell test structures of two different thicknesses were fabricated. The former were analyzed via LBIC and solar simulator measurements, the latter via simulations and solar simulator measurements. We found that the inter-grain diffusion length sinks drastically with higher doping from $29 \pm 7$ to $13 \pm 1 \mu \mathrm{m}$ and that at the same time the passivation quality of grain boundaries and dislocations decreases. The latter is probably due to phosphorous segregation, which could be mitigated using gettering techniques that have so far not been tried on the multi-crystalline thin LPC-Si. The lower material quality of the higher doping leads to a break down in current that cannot be over-compensated by an increase in $V_{O C}$ and $F F$. For the highest doping concentration of $7.0 \cdot 10^{17} / \mathrm{cm}^{3}$ the $V_{O C}$ is again slightly reduced due to the impact of the grain boundaries which would yield global averaged diffusion lengths of 3-4 $\mu \mathrm{m}$ as opposed to the locally found $13 \mu \mathrm{m}$ inside good grains.

Advanced cell concepts for LPC-Si absorbers have to be developed for superstrate (illumination through the glass) operation to make use of the inherent advantages of the LPC$\mathrm{Si}$ technology. Hence, knowledge about the buried and inherently inaccessible glass/LPC-Si interface is of major importance. To get more insight into whether the LPC-Si bulk or the buried interface is limiting the performance at the current state of development, quasi cell test structures were investigated making use of their bifaciality. It was found out that the intermediate layer (IL) sufficiently passivates the LPC$\mathrm{Si} /$ glass interface and that, at this point, more potential to reach higher performance can be expected from improving the bulk. At the current status the bulk lifetimes are around 1-5 $\mu$ s depending on local absorber quality due to the inhomogeneous multi-crystalline nature. The critical buried interface has a surface recombination velocity of around $200 \mathrm{~cm} / \mathrm{s}$. This leads to effective (overall) diffusion lengths $L_{\text {diff }}$ of $24-43 \mu \mathrm{m}$, compared to the values obtained by local LBIC investigations of $(29 \pm 7 \mu \mathrm{m})$ for the same $N_{D}$ of $2.0 \cdot 10^{16} / \mathrm{cm}^{3}$. The two values for $L_{\text {diff }}$ are in excellent agreement and thus show clearly that for low doping concentrations the GBs and dislocations in LPC-Si do not lead to a substantial breakdown of the overall $L_{\text {diff. }}$ This length of $L_{\text {diff }}$ is furthermore enough to tap the full potential of $\sim 15 \mu \mathrm{m}$ thick absorbers. Using thicker absorbers to enhance absorption and hence $J_{S C}$ requires longer diffusion lengths that could be obtained by (i) larger grains or (ii) better passivated grains, which can be reached by gettering techniques [28], improved hydrogen passivation annealing techniques [12,14] or advanced crystallization concepts [9]. This way improving the current highest reported $13.2 \%$ conversion efficiency [11] to above $16 \%$ seems feasible in the future.

\section{ACKNOWLEDGEMENTS}

The authors would like to thank Martina Trahms, Martin Muske, Rutger Schlatmann, Jan Haschke, Miha Filipič and Tim Frijnts for their help in fabrication, characterization and for fruitful discussions. M.B. and M.T. acknowledge financial support from the Slovenian Research Agency (Research Programme P2-0197).

\section{FUNDING}

This research did not receive any specific grant from funding agencies in the public, commercial, or not-for-profit sectors.

\section{REFERENCES}

[1] B. Rech, H. Wagner, Potential of amorphous silicon for solar cells, Appl. Phys. A. 69 (1999) 155-167. doi:10.1007/s003390050986.

[2] D.E. Carlson, C.R. Wronski, Amorphous silicon solar cell, Appl. Phys. Lett. 28 (1976) 671-673. doi:10.1063/1.88617.

[3] H. Sai, T. Matsui, M. Koji, Stabilized 14.0\%-efficient triple-junction thin-film silicon solar cell, Appl. Phys. Lett. 109 (2016) 183506. doi:10.1063/1.4966996.

[4] T. Bearda, I. Gordon, H.S. Radhakrishnan, V. Depauw, K.V. Nieuwenhuysen, M. Xu, L. Tous, M. Filipič, S.K. Jonnak, A. Hajijafarassar, X. Liu, M. Debucquoy, Y. Abdulraheem, J. Szlufcik, J. Poortmans, Thin Epitaxial Silicon Foils Using Porous-Silicon-Based Lift-Off for Photovoltaic Application, MRS Adv. 1 (2016) 32353246. doi:10.1557/adv.2016.314.

[5] R. Cariou, W. Chen, I. Cosme-Bolanos, J.-L. Maurice, M. Foldyna, V. Depauw, G. Patriarche, A. Gaucher, A. Cattoni, I. Massiot, S. Collin, E. Cadel, P. Pareige, P. Roca i Cabarrocas, Ultrathin PECVD epitaxial Si solar cells on glass via low-temperature transfer process, Prog. Photovolt. Res. Appl. 24 (2016) 1075-1084. doi:10.1002/pip.2762.

[6] J. Haschke, D. Amkreutz, B. Rech, Liquid phase crystallized silicon on glass: Technology, material quality and back contacted heterojunction solar cells, Jpn. J. Appl. Phys. 55 (2016) 04EA04. doi:10.7567/JJAP.55.04EA04. 
[7] A.G. Aberle, Thin-film solar cells, Thin Solid Films. 517 (2009) 4706-4710. doi:10.1016/j.tsf.2009.03.056.

[8] J. Haschke, D. Amkreutz, L. Korte, F. Ruske, B. Rech, Towards wafer quality crystalline silicon thin-film solar cells on glass, Sol. Energy Mater. Sol. Cells. 128 (2014) 190-197. doi:10.1016/j.solmat.2014.04.035.

[9] S. Kühnapfel, S. Gall, B. Rech, D. Amkreutz, Towards monocrystalline silicon thin films grown on glass by liquid phase crystallization, Sol. Energy Mater. Sol. Cells. 140 (2015) 86-91. doi:10.1016/j.solmat.2015.03.030.

[10] Moslehi, M.M., Kapur, P., Kramer, J., Rana, V., Seutter, S., Deshpande, A., Stalcup, T., Kommera, S., Ashjaee, J., Calcaterra, A., Grupp, D., World-record $20.6 \%$ efficiency $156 \mathrm{~mm}$ x $156 \mathrm{~mm}$ full-square solar cells using low-cost kerfless ultrathin epitaxial silicon \& porous silicon lift-off technology for industry-leading high-performance smart PV modules., PV Asia Pac. Conf. APVIAPVAP. Vol. 24 (n.d.).

[11] P. Sonntag, N. Preissler, M. Bokalič, M. Trahms, J. Haschke, R. Schlatmann, M. Topic, R. Rech, D. Amkreutz, Silicon Solar Cells on Glass with Power Conversion Efficiency above $13 \%$ at Thickness below 15 Micrometer, Sci. Rep. in press (2017).

[12] N. Preissler, J.A. Töfflinger, O. Gabriel, P. Sonntag, D. Amkreutz, B. Stannowski, B. Rech, R. Schlatmann, Passivation at the interface between liquid-phase crystallized silicon and silicon oxynitride in thin film solar cells, Prog. Photovolt. Res. Appl. (2016) n/a-n/a. doi:10.1002/pip.2852.

[13] J. Dore, S. Varlamov, M.A. Green, Intermediate Layer Development for Laser-Crystallized Thin-Film Silicon Solar Cells on Glass, IEEE J. Photovolt. PP (2014) 1-8. doi:10.1109/JPHOTOV.2014.2361033.

[14] O. Gabriel, T. Frijnts, N. Preissler, D. Amkreutz, S. Calnan, S. Ring, B. Stannowski, B. Rech, R. Schlatmann, Crystalline silicon on glass-interface passivation and absorber material quality, Prog. Photovolt. Res. Appl. (2015) n/a-n/a. doi:10.1002/pip.2707.

[15] M. Vetter, A. Gawlik, J. Plentz, G. Andrä, Carrier Lifetime in Liquid-phase Crystallized Silicon on Glass, Energy Procedia. 92 (2016) 248-254. doi:10.1016/j.egypro.2016.07.067.

[16] J. Haschke, D. Amkreutz, T. Frijnts, S. Kühnapfel, T. Hänel, B. Rech, Influence of Barrier and Doping Type on the Open-circuit Voltage of Liquid Phase Crystallized Silicon Thin-film Solar Cells on Glass, (n.d.). doi:10.1109/JPHOTOV.2015.2412453.

[17] G. Masetti, M. Severi, S. Solmi, Modeling of carrier mobility against carrier concentration in arsenic-, phosphorus-, and boron-doped silicon, IEEE Trans. Electron Devices. 30 (1983) 764-769. doi:10.1109/TED.1983.21207.

[18] IEC-norm, [IEC60904-3:2008], n.d.
[19] M. Bokalič, M. Topič, Light beam characterisation of LBIC apparatus and selected complementary applications, in: Proc 46th MIDEM Conf., 2010: pp. 233-237.

[20] M. Filipič, Z.C. Holman, F. Smole, S.D. Wolf, C. Ballif, M. Topič, Analysis of lateral transport through the inversion layer in amorphous silicon/crystalline silicon heterojunction solar cells, J. Appl. Phys. 114 (2013) 74504. doi:10.1063/1.4818709.

[21] P. Sonntag, J. Haschke, S. Kühnapfel, T. Frijnts, D. Amkreutz, B. Rech, Interdigitated back-contact heterojunction solar cell concept for liquid phase crystallized thin-film silicon on glass, Prog. Photovolt. Res. Appl. 24 (2016) 716-724. doi:10.1002/pip. 2725.

[22] C. Ulbrich, A. Gerber, K. Hermans, A. Lambertz, U. Rau, Analysis of short circuit current gains by an antireflective textured cover on silicon thin film solar cells, Prog. Photovolt. Res. Appl. 21 (2013) 1672-1681. doi:10.1002/pip.2249.

[23] P. Sonntag, M. Bokalič, M. Filipič, T. Frijnts, D. Amkreutz, M. Topič, B. Rech, Analysis of Local Minority Carrier Diffusion Lengths in Liquid-Phase Crystallized Silicon Thin-Film Solar Cells, IEEE J. Photovolt. PP (2016) 1-5. doi:10.1109/JPHOTOV.2016.2615680.

[24] A.G. Aberle, P.I. Widenborg, Crystalline Silicon ThinFilm Solar Cells via High-Temperature and Intermediate-Temperature Approaches, in: A. Luque, S. Hegedus (Eds.), Handb. Photovolt. Sci. Eng., John Wiley \& Sons, Ltd, 2010: pp. 452-486.

[25] M. Mews, T.F. Schulze, N. Mingirulli, L. Korte, Hydrogen plasma treatments for passivation of amorphous-crystalline silicon-heterojunctions on surfaces promoting epitaxy, Appl. Phys. Lett. 102 (2013) 122106. doi:10.1063/1.4798292.

[26] J.H. Rose, R. Gronsky, Scanning transmission electron microscope microanalytical study of phosphorus segregation at grain boundaries in thin-film silicon, Appl. Phys. Lett. 41 (1982) 993-995. doi:10.1063/1.93368.

[27] M.M. Mandurah, K.C. Saraswat, C.R. Helms, T.I. Kamins, Dopant segregation in polycrystalline silicon, J. Appl. Phys. 51 (1980) 5755-5763. doi:10.1063/1.327582.

[28] A. Bentzen, A. Holt, R. Kopecek, G. Stokkan, J.S. Christensen, B.G. Svensson, Gettering of transition metal impurities during phosphorus emitter diffusion in multicrystalline silicon solar cell processing, J. Appl. Phys. 99 (2006) 93509. doi:10.1063/1.2194387. 